\title{
Evaluation of Hepatoprotective Effect of Vanillin in Isoniazid-Rifampicin Induced Hepatocellular Damage
}

\author{
Rashint Tiwari, Ayushi Chourasia, Aman Chaturvedi, Aditya Ganeshpurkar*, Nazneen Dubey
}

\section{Rashint Tiwari, Ayushi Chourasia, Aman Chaturvedi, Aditya Ganeshpurkar*, Nazneen Dubey}

Shri Ram Institute of Technology-Pharmacy, Jabalpur, Madhya Pradesh, INDIA.

\section{Correspondence}

Dr. Aditya Ganeshpurkar

Assistant Professor, Shri Ram Institute of Technology- Pharmacy, Jabalpur-482002, Madhya Pradesh, INDIA.

Phone no: +91 0761-4041266

E-mail: adityaganeshpurkar@gmail.com

History

- Submission Date: 29-05-2020;

- Review completed: 02-06-2020;

- Accepted Date: 18-06-2020.

DOI : 10.5530/fra.2020.1.8

Article Available online

http://www.antiox.org

Copyright

(C) 2020 Phcog.Net. This is an openaccess article distributed under the terms of the Creative Commons Attribution 4.0 International license.

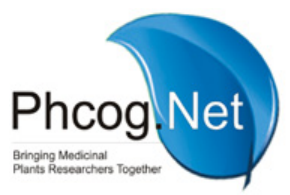

\begin{abstract}
Objectives: Natural products are greatly acknowledged for antioxidant and hepatoprotective effects. Vanillin has been studied for radical scavenging effect. The aim of this study was to examine hepatoprotective effect of vanillin against isoniazid and rifampicin induced liver damage in rats. Methods: Wistar rats were used in present study. All the animals study protocols were duly approved by Institutional Animal Ethics Committee of the Institute. Hepatotoxicity was induced by administration of against isoniazid $(50 \mathrm{mg} / \mathrm{kg}$ ) and rifampicin $(100 \mathrm{mg} / \mathrm{kg})$ for 14 days. Vanillin was used in the dose of 50,100 and $200 \mathrm{mg} / \mathrm{kg}$ body weight. At the end of study blood was collected and biochemical studies were performed to assess antioxidant status. Results: Oral administration of vanillin at test doses (50, 100 and $200 \mathrm{mg} /$ $\mathrm{kg}$ body weight) resulted in restoration of AST, ALT and ALP. There was a notable decrease in production of SOD and catalase. Conclusion: In the present study, vanillin demonstrated a notable hepatoprotective effect. The protective efficacy of vanillin is possibly because of radical scavenging and antioxidant property.

Key words: Liver, Toxicity, Antitubercular, Peroxidation, Vanillin.
\end{abstract}

\section{INTRODUCTION}

Drug induced liver injury are one of the leading cause of liver damage worldwide. ${ }^{1}$ Incidence, progression, and outcomes of NAFLD and nonalcoholic steatohepatitis (NASH Long term use of some drugs adversely influences liver by inducing oxidative stress which arises due to imbalance between antioxidant and pro-oxidant system. ${ }^{2}$ Such oxidative stress for the long period of time may be predispose to fatty liver which further leads to fibrosis, inflammation and cirrhosis. ${ }^{3}$ Isoniazid and rifampicin are the two important classes of anti-tubercular drugs used widely for the management of tuberculosis. ${ }^{4}$ However, these drugs suffer from a serious adverse effect of hepatocellular damage. ${ }^{5}$ Phytochemicals are the chemicals that are produced by plants as a consequence of a regular metabolism. They are widely distributed in plant kingdom. About 8000 polyphenolic structures are known out of which nearly 4000 are identified. ${ }^{6}$ Polyphenols are the secondary metabolites that are widely distributed in plant kingdom. They have antioxidant property which helps them to provide protection against oxidative crisis caused by reactive oxygen species. Evidences provide an insight towards their biological role to arbitrator the antioxidant activity. ${ }^{7,8}$ Vanilla (Vanilla planifolia L.) is one of the most popular aromas worldwide, which has given a unique taste to bakery products and confectionary. Vanilla seems to be originated from Central America and Mexico. ${ }^{9}$
Natural vanilla is a complex blend of flavors from the cured pods of Vanilla planifolia and Vanilla tahitensis. ${ }^{10}$ Vanillin is the chief component which constitutes of $1-2 \% \%$ of vanilla extract. ${ }^{11}$ It is a white powder crystalline in nature. It is a sweet substance with vanilla flavor. Chemically, vanillin is a phenol 'substituted' with an aldehyde and methoxy group. Vanillin has been studied for various biological effects which includes analgesic, ${ }^{12}$ neuro-protective $\mathrm{p}$-ERK ${ }^{13}$ and antimutagenic $\mathrm{c}^{14,15}$ as well as mutagenesis, induced in Escherichia coli by $\mathrm{N}$-methyl-N-nitrosourea (MNU effect. In a study, vanillin caused a significant inhibition on oxygen radical absorbance capacity assay and in the oxidative hemolysis inhibition assay. ${ }^{16}$ Similarly, vanillin afforded a significant protection against lipid peroxidation and protein oxidation in mitochondria of hepatocytes sensitized by methylene blue and light. ${ }^{17}$ The present study was aimed to determine the protective effect of vanillin on isoniazid-rifampicin induced hepato-cellular damage in rats.

\section{Experimental \\ Animals}

Laboratory-bred Wistar rats (180-200 g) of either sex were housed in polypropylene cages, maintained under standardized conditions (12 h light/dark cycles, $28 \pm 2^{\circ} \mathrm{C}$ ) were used in the study. Animals were provided with standard pellet food and had

Cite this article: Tiwari R, Chourasia A, Chaturvedi A, Ganeshpurkar A, Dubey N. Evaluation of Hepatoprotective Effect of Vanillin in Isoniazid-Rifampicin Induced Hepatocellular Damage. Free Radicals and Antioxidants. 2020;10(1):42-6. 
free access to drinking water. All the animal study protocols were duly approved by Institutional Animal Ethics Committee.

\section{Selection of dose}

The aqueous solution of vanillin in doses of 50, 100 and $200 \mathrm{mg} / \mathrm{kg}$ body weight was freshly prepared and administered orally. ${ }^{18}$

\section{Animal group and dosing}

Animals were divided into six groups with six animals in each group.

Group, I Normal Saline $2 \mathrm{ml} / \mathrm{kg}$

Group II Rifampicin (50mg/kg) and Isoniazid (100mg/kg)

Group III Rifampicin $(50 \mathrm{mg} / \mathrm{kg})$ and Isoniazid $(100 \mathrm{mg} / \mathrm{kg})+$ Silymarin

Group IV Rifampicin $(50 \mathrm{mg} / \mathrm{kg})$ and Isoniazid $(100 \mathrm{mg} / \mathrm{kg})+$ Vanillin $50 \mathrm{mg} / \mathrm{kg}$

Group V Rifampicin (50mg/kg) and Isoniazid (100mg/kg) + Vanillin $100 \mathrm{mg} / \mathrm{kg}$

Group VIRifampicin $(50 \mathrm{mg} / \mathrm{kg})$ and Isoniazid $(100 \mathrm{mg} / \mathrm{kg})+$ Vanillin $200 \mathrm{mg} / \mathrm{kg}$

On the $14^{\text {th }}$ day blood was withdrawn by retro orbital puncture for the estimation of biochemical parameters. After that, animals were sacrificed under ether anaesthesia. The liver was collected, washed and used for histopathological studies.

\section{Biochemical analysis}

Blood samples were collected into the epindrop tubes and centrifuged for $10 \mathrm{~min}$ at $7000 \mathrm{rpm}$ using micro-centrifuge to separate the serum. The levels of serum glutamic oxaloacetic transaminase (SGOT/AST), serum glutamic-pyruvic transaminase (SGPT/ALT) serum alkaline phosphatase (SALP) were estimated using commercial kits (Span Diagnostics, India).

\section{Antioxidant enzymes}

\section{Superoxide dismutase assay}

Superoxide dismutase (SOD) activity in liver homogenate was determined according to the method of Minami and Yoshikawa. ${ }^{19}$ The method was based on the generation of superoxide anions by pyrogallol autoxidation, detection of generated superoxide anions by nitro blue tetrazolium (NBT) formazan colour development and measurement of the amount of generated superoxide anions scavenged by SOD (the inhibitory level of formazan colour development). The liver homogenate was centrifuged to $10000 \mathrm{rpm}$ for $15 \mathrm{~min}$ at $4^{\circ} \mathrm{C}$. To $0.25 \mathrm{ml}$ of supernatant, $0.5 \mathrm{ml}$ of tris cacodylic buffer, $0.1 \mathrm{ml}$ of $16 \%$ triton $\mathrm{x}-100$ and $0.25 \mathrm{ml} \mathrm{NBT}$ were

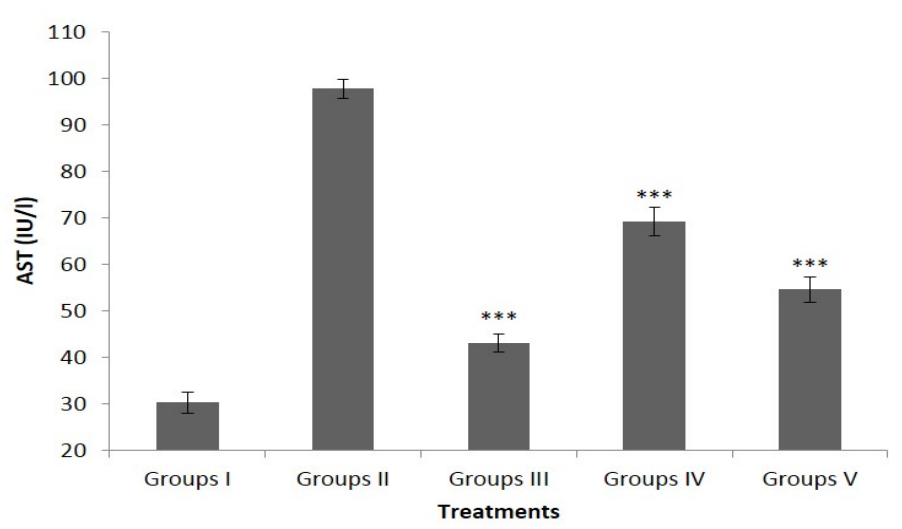

Figure 1: Effect of vanillin administration on AST levels in isoniazid-rifampicin treated rats. Results are given as mean \pm SEM of six animals in each group. added. The reaction was started by the addition of $0.01 \mathrm{ml}$ diluted pyrogallol. Incubation was maintained for $5 \mathrm{~min}$ at $37^{\circ} \mathrm{C}$. The reaction was stopped by the addition of $0.3 \mathrm{ml}$ of $2 \mathrm{M}$ formic acid. The formazan colour developed was determined spectrophotometrically at wavelength $430 \mathrm{~nm}$. Enzymatic activity was expressed as $\mu \mathrm{g} / \mathrm{gm}$ of tissue.

\section{Catalase activity}

The catalase activity was measured according to method of Sinha. ${ }^{20} 0.1 \mathrm{ml}$ of liver homogenate was mixed with $1.0 \mathrm{ml}$ of $0.01 \mathrm{M}$ phosphate buffer ( $\mathrm{pH} 7.4$ ) and incubated with $0.4 \mathrm{ml}$ of $0.2 \mathrm{M} \mathrm{H}_{2} \mathrm{O}_{2}$ at $37^{\circ} \mathrm{C}$ accurately for $1.0 \mathrm{~min}$ and reaction was stopped with $2.0 \mathrm{ml}$ of $5 \%$ potassium dichromate (1:3 with glacial acetic acid). Further the samples were incubated in boiling water bath for $15 \mathrm{~min}$. Tubes were centrifuged at $5000 \mathrm{rpm}$ for $15 \mathrm{~min}$ and supernatant was used to quantify the amount of $\mathrm{H}_{2} \mathrm{O}_{2}$ to calculate catalase activity at $570 \mathrm{~nm}$. One unit represents 1.0 $\mu \mathrm{mole}$ of $\mathrm{H}_{2} \mathrm{O}_{2}$ consumed $/ \mathrm{min} / \mathrm{mg}$ protein

\section{Statistical analysis}

The results were expressed as mean \pm SEM. Statistical analysis was carried out by using One way ANOVA followed by Dennett's' test and $p<0.01$, $p<0.001$ was considered significant.

\section{RESULTS}

\section{Effect on marker enzyme levels}

Rats treated with isoniazid and rifampicin developed a significant hepatic damage observed as elevated serum levels of hepato-specific enzymes such as ALT, AST, ALP and decreased level of total protein when compared to normal control. In the present study, there was restoration of antioxidant enzyme level (AST, ALP and ALT) animals of the group IV to VI treated (50, 100 and $200 \mathrm{mg} / \mathrm{kg}$ ) with vanillin (Figure 1-3). Pre-treatment with silymarin also afforded a significant protection against isoniazid and rifampicin induced toxicity in liver.

\section{Superoxide dismutase and Catalase}

The administration of isoniazid and rifampicin to animals resulted in a decrement in levels of SOD. But, treatment with vanillin (50, 100 and $200 \mathrm{mg} / \mathrm{kg})$ resulted in a significant $(p<0.001)$ increment in SOD levels as compared to toxic control group (Group II). Congruently, the administration of vanillin (50,100 and $200 \mathrm{mg} / \mathrm{kg}$ ) in animals resulted in significant $(p<0.001)$ increment in the levels of SOD and catalase. The administration of silymarin also resulted in decrement in the levels of SOD and catalase levels (Figure 4-5).

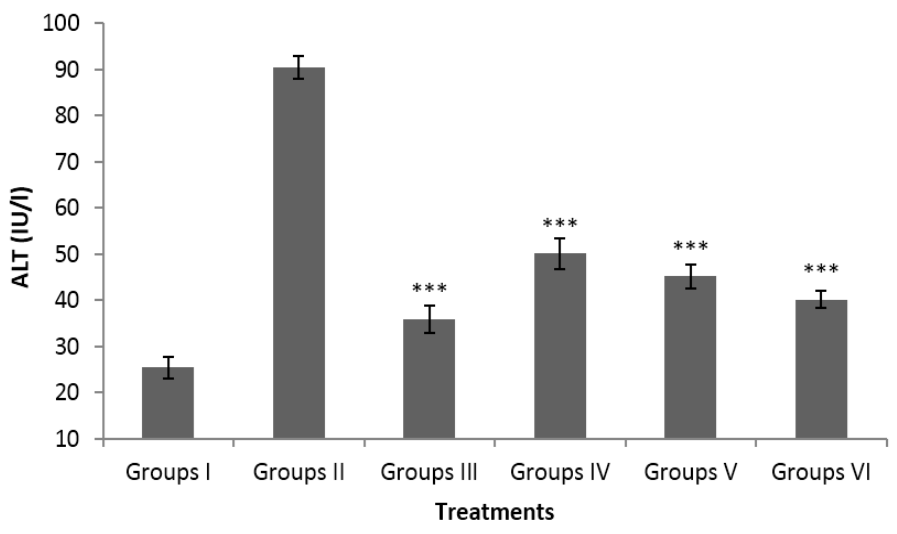

Figure 2: Effect of vanillin administration on ALT levels in isoniazid-rifampicin treated rats. Results are given as mean \pm SEM of six animals in each group. 


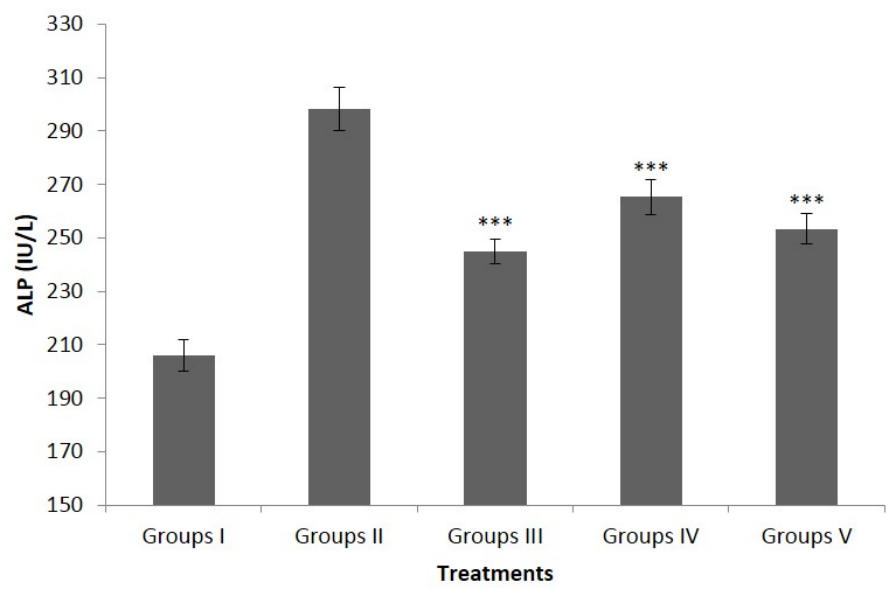

Figure 3: Effect of vanillin administration on ALP levels in isoniazid-rifampicin treated rats. Results are given as mean \pm SEM of six animals in each group.

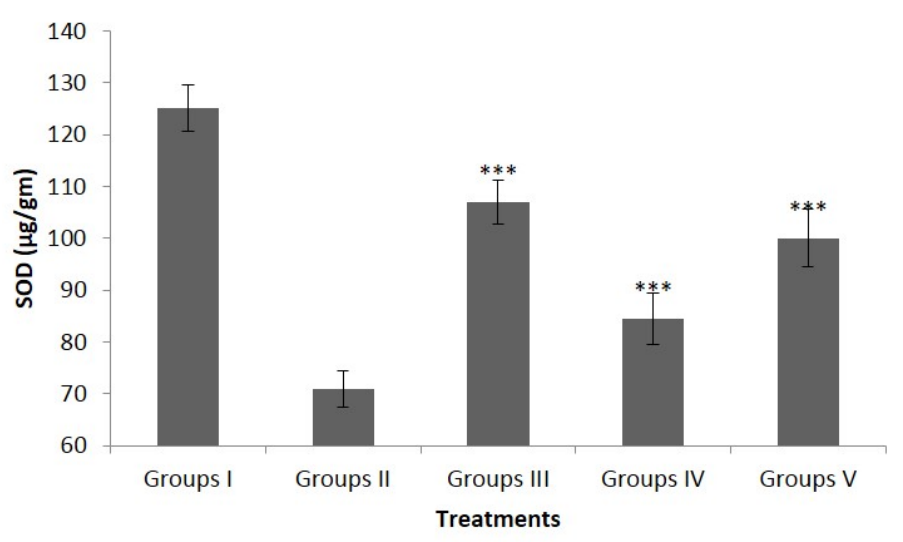

Figure 4: Effect of vanillin administration on SOD levels in isoniazid-rifampicin treated rats. Results are given as mean \pm SEM of six animals in each group.

\section{DISCUSSION}

Isoniazid is a gold standard anti-mycobacterial agent used in the management of tuberculosis world-wide. The metabolic products arising due to biotransformation of isoniazid (hydrazine, acetyl-hydrazine) are responsible to predispose liver toxicity. This is due to formation of covalent adducts with liver macromolecules. These adducts form covalent bonds with many lysine residues of hepatic proteins ${ }^{5}$ Further auto oxidation of isoniazid is also responsible for production of free radicals. ${ }^{21}$ Due to this type of biological stress, there is generation of anti-isoniazid antibodies. ${ }^{22}$ Rifampicin is another anti-tubercular agent used in the management of tuberculosis. The experimental studies have showed that co-administration of rifampicin with isoniazid resulted in a more significant liver injury. ${ }^{23}$ The remedies to control and reverse hepatotoxicity include use of radical-scavengers and antioxidants. Antioxidants are known to revert oxidative stress inside the cell. The various antioxidants from natural sources have been experimentally tested for their hepatoprotective potential. They include rutin, ${ }^{24}$ hesperidin, ${ }^{25}$ resveratrol, ${ }^{26}$ silymarin, ${ }^{27}$ naringenin ${ }^{28}$ etc.

The aim of present study was to evaluate protective effect of vanillin on isoniazid-rifampicin induced hepatotoxicity in rats. Vanillin was administered to animals in the dose of 50,100 and $200 \mathrm{mg} / \mathrm{kg}$. The administration of vanillin resulted in restoration of antioxidant

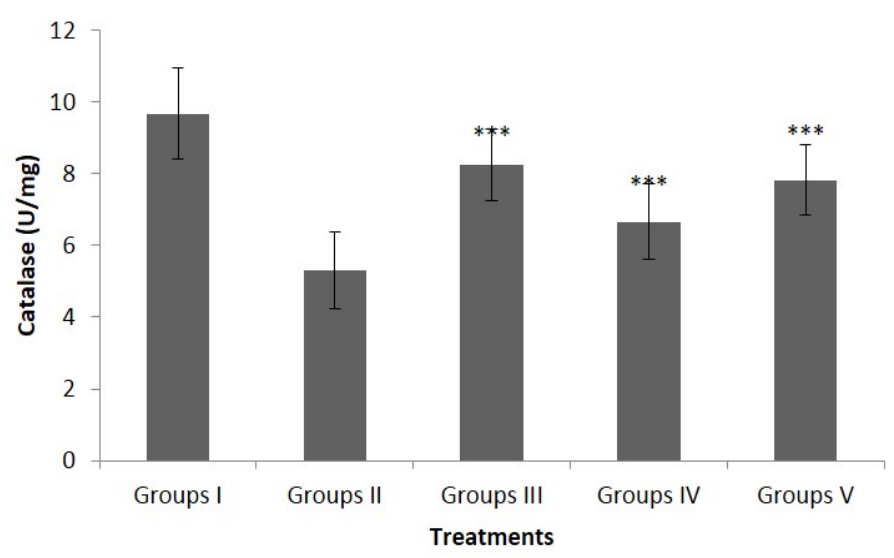

Figure 5: Effect of vanillin administration on catalase levels in isoniazid-rifampicin treated rats. Results are given as mean \pm SEM of six animals in each group.

enzyme level (AST, ALP and ALT) animals of the group IV to VI. The administration of vanillin $(50,100$ and $200 \mathrm{mg} / \mathrm{kg})$ in animals resulted in notable increment in the levels of SOD, catalase and bilirubin. There was a decrement in lipid peroxidation near to normal levels. The bilirubin and serum ALP are closely allied with the functioning of liver cells. An increased level of serum bilirubin and ALP point to biliary pressure. ${ }^{29}$ In the present study, administration of vanillin to animals resulted in a notable decrement in bilirubin and ALP.

There is a well-developed defence system developed in multicellular eukaryotic organisms which aids in neutralization of damage induced by free radicals. The antioxidant enzymes viz. SOD and catalase work proficiently to prevent free radical induced damage. ${ }^{30}$ However, isoniazid and rifampicin alter the activity of this system by producing reactive species, which probably interact with enzymes making them nonfunctional and highlight hepatic damage. The treatment with vanillin resulted in a significant increase in these enzyme levels which highlight their ability to scavenge reactive oxygen species. An increased production of lipid-peroxides represents the damage to cells. The increment in raised free radical production damages cell morphology and results in oxidative stress induced cell damage. ${ }^{31}$ The present study revealed a reduction in free radical production.

Liver is a vital organ of the body. Various processes like calorie-genesis, enzyme production, detoxification, drug metabolism and many more processes take place inside the liver. The liver damage is associated with altered metabolism which can lead to progression cirrhosis. Therefore, the drugs/ chemicals which protect the hepatocellular damage are of extreme interest. The outcomes of the present studies testified the beneficial effect of vanillin administration on rat model.

Based on the above observations, it can be summarized that vanillin treatment resulted in restoration of liver antioxidant status and antioxidant enzymes. It caused a decrement in lipid peroxidation. The cellular integrity of hepatocytes was guarded by vanillin. Thus, vanillin can be regarded as a potential antioxidant and can be tested for molecular mechanism for hepatoprotective effects.

\section{CONCLUSION}

The outcomes of the present study highlight the role of vanillin in preventing the harmful effects of isoniazid-rifampicin on rats. These promising results can help in development of antioxidant and hepatoprotective nutraceuticals. 


\section{ACKNOWLEDGEMENT}

Authors are thankful to Principal, Shri Ram Institute of TechnologyPharmacy for providing necessary facilities for performing the studies.

\section{CONFLICT OF INTEREST}

The authors declare no conflict of interest.

\section{ABBREVIATIONS}

ALP: Alkaline phosphatase; ALT: Alanine transaminase; AST: Aspartate transaminase; CAT: Catalase; $\mathrm{H}_{2} \mathrm{O}_{2}$ : Hydrogen peroxide; SOD: Superoxide dismutase.

\section{REFERENCES}

1. Younossi ZM, Koenig AB, Abdelatif D, Fazel Y, Henry L, Wymer M. Global epidemiology of nonalcoholic fatty liver disease-Meta-analytic assessment of prevalence, incidence and outcomes. Hepatology. 2016;64(1):73-84.

2. Li S, Tan HY, Wang N, Zhang ZJ, Lao L, Wong CW. The role of oxidative stress and antioxidants in liver diseases. Int J Mol Sci. 2015;16(11):26087-124.

3. Bessone F, Dirchwolf M, Rodil MA, Razori MV, Roma MG. Drug-induced liver injury in the context of nonalcoholic fatty liver disease- a physiopathological and clinical integrated view. Aliment Pharmacol Ther. 2018;48(9):892-913.

4. Zumla A, Chakaya J, Centis R, D'Ambrosio L, Mwaba P, Bates M. Tuberculosis treatment and management-an update on treatment regimens, trials, new drugs and adjunct therapies. Lancet Respir Med. 2015;3(3):220-34.

5. Biswas SSA, Bishu D, Dhali GK, Chowdhury A, Santra A. Anti-TB Drugs, Isoniazid and Rifampicin Produce Hepatic Fibrosis Through a Oxidative StressDependent Mechanism. J Clin Exp Hepatol. 2015;5:S80-5.

6. Karunaweera N, Raju R, Gyengesi E, Münch G. Plant polyphenols as inhibitors of NF-KB induced cytokine production-a potential anti-inflammatory treatment for Alzheimer's disease?. Front Mol Neurosci 2015;8:24.

7. Carrasco-Pozo C. Protection by Polyphenols Against Mitochondrial Damage and Cytotoxicity. In: Polyphenols in Human Health and Disease. 2013;731-46.

8. Castelli V, Grassi D, Bocale R, D'Angelo M, Antonosante A, Cimini A. Diet and Brain Health: Which Role for Polyphenols?. Curr Pharm Des. 2017;24(2):227-38.

9. Zhang H, Tsao R. Dietary polyphenols, oxidative stress and antioxidant and antiinflammatory effects. Curr Opin Food Sci. 2016;8:33-42.

10. Gallage NJ, Møller BL. Vanillin-bioconversion and bioengineering of the most popular plant flavor and its de novo biosynthesis in the vanilla orchid. Mol. Plant. 2015;8(1):40-57.

11. Zhang Y, Mo L, Chen F, Lu M, Dong W, Wang $Q$. Optimized production of vanillin from green vanilla pods by enzyme-assisted extraction combined with prefreezing and thawing. Molecules. 2014;19(2):2181-98.

12. Beaudry F, Ross A, Lema PP, Vachon P. Pharmacokinetics of vanillin and its effects on mechanical hypersensitivity in a rat model of neuropathic pain Phyther Res. 2010;24(4):525-30.

13. Dhanalakshmi C, Manivasagam T, Nataraj J, Justin TA, Essa MM Neurosupportive Role of Vanillin, a Natural Phenolic Compound, on Rotenone Induced Neurotoxicity in SH-SY5Y Neuroblastoma Cells. Evidence-based
Complement Altern Med. 2015;626028.

14. Takahashi K, Sekiguchi M, Kawazoe Y. Effects of vanillin and o-vanillin on induction of DNA-repair networks: Modulation of mutagenesis in Escherichia coli. Mutat Res - Fundam Mol Mech Mutagen. 1990;230(2):127-34

15. Shaughnessy DT, Schaaper RM, Umbach DM, DeMarini DM. Inhibition of spontaneous mutagenesis by vanillin and cinnamaldehyde in Escherichia coli: Dependence on recombinational repair. Mutat Res - Fundam Mol Mech Mutagen. 2006;602(1-2):54-64.

16. Tai A, Sawano T, Yazama F, Ito $H$. Evaluation of antioxidant activity of vanillin by using multiple antioxidant assays. Biochim Biophys Acta - Gen Subj 2011;1810(2):170-7.

17. Kamat JP, Ghosh A, Devasagayam TPA. Vanillin as an antioxidant in rat liver mitochondria: Inhibition of protein oxidation and lipid peroxidation induced by photosensitization. Mol Cell Biochem. 2000;209(1-2):47-53.

18. Al-Asmari A, Al-Shahrani H, Al-Masri N, Al-Faraidi A, Elfaki I, Arshaduddin M Vanillin abrogates ethanol induced gastric injury in rats via modulation of gastric secretion, oxidative stress and inflammation. Toxicol Reports. 2016;3:105-13.

19. Masayasu M, Hiroshi Y. A simplified assay method of superoxide dismutase activity for clinical use. Clin Chim Acta. 1979;92(3):337-42.

20. Sinha AK. Colorimetric assay of catalase. Anal Biochem. 1972;47(2):389-94.

21. Meng X, Maggs JL, Usui T, Whitaker P, French NS, Naisbitt DJ. Auto-oxidation of isoniazid leads to isonicotinic-lysine adducts on human serum albumin. Chem Res Toxicol. 2015;28(1):51-8

22. Metushi IG, Sanders C, Group ALS, Lee WM, Uetrecht J. Detection of antiisoniazid and anti-cytochrome P450 antibodies in patients with isoniazidinduced liver failure. Hepatology. 2014;59(3):1084-93.

23. Baskaran UL, Sabina EP. Clinical and experimental research in antituberculosis drug-induced hepatotoxicity: A review. J Integr Med. 2017; 15(1):27-36.

24. Abdel-Ghaffar O, Mahmoud ST, Said AA, Sanad FAY. Hepatoprotective effect of rutin against oxidative stress of isoniazid in albino rats. Int $\mathrm{J}$ Pharmacol. 2017;13(6):516-28

25. Zhang G, Zhu J, Zhou Y, Wei Y, Xi L, Qin H. Hesperidin alleviates oxidative stress and upregulates the multidrug resistance protein 2 in isoniazid and rifampicininduced liver injury in rats. J Biochem Mol Toxicol. 2016;30(7):342-9.

26. Nicoletti NF, Rodrigues-Junior V, Santos JAA, Leite CE, Dias ACO, Batista JEL. Protective effects of resveratrol on hepatotoxicity induced by isoniazid and rifampicin via SIRT1 modulation. J Nat Prod. 2014;77(10):2190-5.

27. Sabina EP, Peter SJ, Geetha A. A comparison of hepatoprotective activity of Bacoside to Silymarin treatment against a combined Isoniazid and Rifampininduced hepatotoxicity in female Wistar rats. J Histotechnol. 2019;42(3):128-36.

28. Wang $\mathrm{C}$, Fan RQ, Zhang YX, Nie H, Li K. Naringenin protects against isoniazidand rifampicin-induced apoptosis in hepatic injury. World J Gastroenterol. 2016;22(44):9775

29. Kumar R, Bhatia V, Khanal S, Sreenivas V, Gupta SD, Panda SK. Antituberculosis therapy-induced acute liver failure: Magnitude, profile, prognosis and predictors of outcome. Hepatology. 2010;51(5):1665-74

30. Ighodaro OM, Akinloye OA. First line defence antioxidants-superoxide dismutase (SOD), catalase (CAT) and glutathione peroxidase (GPX): Thei fundamental role in the entire antioxidant defence grid. Alexandria J Med. 2018;54(4):287-93

31. Ahmadinejad F, Geir MS, Hashemzadeh-Chaleshtori M, Bidkhori G, Jami MS. Molecular mechanisms behind free radical scavengers function against oxidative stress. Antioxidants. 2017;6(3):51. 


\section{GRAPHICAL ABSTRACT}

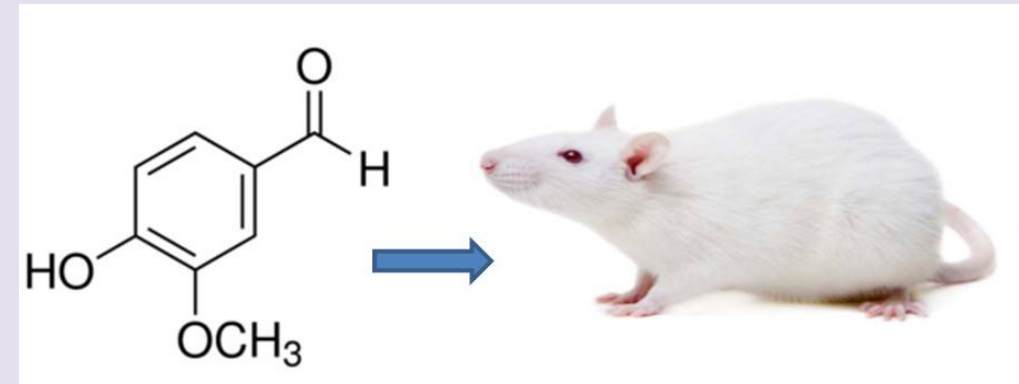

Vanillin treatment in rats

\section{SUMMARY}

The present work establishes hepatoprotective effect of vanillin. More studies at molecular level might be helpful in estabilishing molecular mechanism of action.

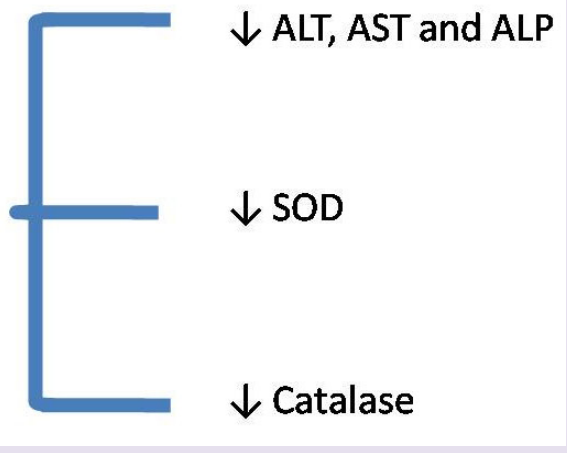

\section{ABOUT AUTHORS}

Mr. Rashint Tiwari holds Post Graduate degree in Pharmacy (M.Pharm) with specialization in Pharmacology. His research interest includes evaluation of bioactive- compounds for organ protective effects.

Cite this article: Tiwari R, Chourasia A, Chaturvedi A, Ganeshpurkar A, Dubey N. Evaluation of Hepatoprotective Effect of Vanillin in Isoniazid-Rifampicin Induced Hepato-cellular Damage. Free Radicals and Antioxidants. 2020;10(1):42-6. 